\title{
Total Laboratory Automation and Three Shifts Reduce Turnaround Time of Cerebrospinal Fluid Culture Results in the Chinese Clinical Microbiology Laboratory
}

Weili Zhang, Siying Wu, Jin Deng, Quanfeng Liao, Ya Liu, Li Xiong, Ling Shu, Yu Yuan, Yuling Xiao, Ying Ma, Mei Kang, Dongdong $\mathrm{Li}^{*}$ and Yi Xie*

Department of Laboratory Medicine, West China Hospital of Sichuan University, Chengdu, China

OPEN ACCESS

Edited by:

Alexandra Aubry,

Sorbonne Universités, France

Reviewed by:

Olivier Dauwalder,

Hospices Civils de Lyon, France Alexandre Godmer,

Assistance Publique Hopitaux De

Paris, France

*Correspondence:

Dongdong Li

jiangxili1219@163.com

YiXie

xie_yi_77@163.com

Specialty section:

This article was submitted to

Clinical Microbiology,

a section of the journal

Frontiers in Cellular and

Infection Microbiology

Received: 01 September 2021 Accepted: 15 November 2021

Published: 02 December 2021

Citation:

Zhang W, Wu S, Deng J, Liao Q, Liu Y, Xiong L, Shu L, Yuan Y, Xiao Y,

Ma Y, Kang M, Li D and Xie Y (2021)

Total Laboratory Automation and

Three Shifts Reduce Turnaround

Time of Cerebrospinal Fluid Culture

Results in the Chinese Clinical

Microbiology Laboratory.

Front. Cell. Infect. Microbiol. 11:765504.

doi: 10.3389/fcimb.2021.765504
Background: Total laboratory automation (TLA) has the potential to reduce specimen processing time, optimize workflow, and decrease turnaround time (TAT). The purpose of this research is to investigate whether the TAT of our laboratory has changed since the adoption of TLA, as well as to optimize laboratory workflow, improve laboratory testing efficiency, and provide better services of clinical diagnosis and treatment.

Materials and Methods: Laboratory data was extracted from our laboratory information system in two 6-month periods: pre-TLA (July to December 2019) and post-TLA (July to December 2020), respectively.

Results: The median TAT for positive cultures decreased significantly from pre-TLA to post-TLA (65.93 vs 63.53, $P<0.001)$. For different types of cultures, The TAT of CSF changed the most (86.76 vs 64.30, $P=0.007$ ), followed by sputum (64.38 vs 61.41, $P<0.001)$, urine (52.10 vs 49,57, $P<0.001)$, blood (68.49 vs 66.60, $P<0.001)$. For Ascites and Pleural fluid, there was no significant difference $(P>0.05)$. Further analysis found that the incidence of broth growth only for pre-TLA was $12.4 \%$ (14/133), while for post-TLA, it was $3.4 \%$ (4/119). The difference was statistically significant $(P=0.01)$. The common isolates from CSF samples were Cryptococcus neoformans, coagulase-negative Staphylococcus, Acinetobacter baumannii, and Klebsiella pneumonia.

Conclusion: Using TLA and setting up three shifts shortened the TAT of our clinical microbiology laboratory, especially for CSF samples.

Keywords: total laboratory automation, turnaround time, clinical microbiology, Kiestra, CSF

\section{BACKGROUND}

Automation has become increasingly common in clinical laboratories, but not yet in clinical microbiology laboratories. There are many possible reasons, such as diversity of clinical samples, culture media, and culture conditions (Dauwalder et al., 2016). The traditional manual operation of the clinical microbiology laboratory can be time-consuming and inefficient. For example, around 
45 manual steps are required from specimen receiving to final report (Croxatto et al., 2015). Now the blood culture instrument adopts a continuous monitoring function to shorten the time of bloodstream infection diagnosis and treatment, which inspires people to explore the development of an automated system of clinical microbiology laboratories. Total laboratory automation (TLA) in clinical microbiology laboratories is defined as instrumentation that automates the bacteriology processes from specimen receiving to discarding plates (Thomson and McElvania, 2019). There are two widely used automation systems available: BD Kiestra Total Laboratory Automation (TLA; Becton-Dickinson, Sparks, MD) and Copan WASPLab (Copan Diagnostics Inc, Murrieta, CA) (Dauwalder et al., 2016; Croxatto et al., 2016; Thomson and McElvania, 2019).

Our clinical microbiology laboratory implemented the BD Kiestra TLA platform from March 2020. To make the best out of the advantages of TLA continuous reception, regular photography, and reading images via the computer screen, we have adjusted the schedule of working shifts from one type (day shift) to three types (morning shift, day shift, and night shift). The purpose of this research is to investigate whether the turnaround time (TAT) of our laboratory has changed since the adoption of TLA, as well as to optimize laboratory workflow, improve laboratory testing efficiency, and provide better services for clinical diagnosis and treatment.

\section{MATERIALS AND METHODS}

This study was performed in West China Hospital of Sichuan University with a total of 4300 beds. The clinical microbiology laboratory performs approximately 23000 cultures per month.

Before and after using TLA, our inoculation process for each type of specimen was the same, that was, using the same material plates (Antubio, Zhengzhou, China) and the same amount of inoculation (for example, for urine, inoculating 10ul sample on the blood plate and MacConkey plate). The diameter of plates varied from $7 \mathrm{~cm}$ before TLA to $9 \mathrm{~cm}$ after TLA to meet the requirements of Kiestra TLA.

\section{Pre-TLA Process}

Inpatient and outpatient specimens were received for processing throughout the day. For the conventional plating technique, specimens were manually streaked using corresponding agar plates and manually placed into incubators. Plates were incubated overnight, and then manually removed from the incubators and read during the day shift (8 a.m. to 4 p.m.). The main work of the plates screening post was to manually remove the plates from the incubator for reading, perform subcultures, identification (ID), and antimicrobial susceptibility testing (AST) for the culture-positive specimens, and report the culture-negative specimens. The reports review post was to report AST results and retain bacteria. The blood cultures post was required to grade reports of positive blood cultures and report negative results. The day shift pre-TLA of our lab was elaborated in Figure 1.

\section{Post-TLA Process and Three Shifts}

BD Kiestra TLA was implemented in March 2020. With TLA, specimens were inoculated onto corresponding agar plates by the fully automated InoqulA+ system, and plates were transported via conveyor tracks (ProceedA) to the incubators (ReadA Compact). Digital images of the plates were taken at the user-specified time (every $6 \mathrm{~h}$ after reception) and were interpreted by technologists throughout the three shifts (morning shift: 7 a.m.-3 p.m., day shift: 8 a.m.-4 p.m., night shift: 4 p.m.-10 p.m.) whenever images became available. The staff in morning shift were mainly responsible for the ID and AST of the positive cultures with a single pure colony cultured from cerebrospinal fluid (CSF), pleural fluid, ascites, urine, and other specimens, as well as the subcultures isolated from the positive cultures of the previous day. The staff in day shift was responsible for the review of AST reports, the subculture, and identification of sputum, swabs, feces, and other specimens. The night shift started the second round of plates screening and subsequent processing at 4 p.m. and then the second round of

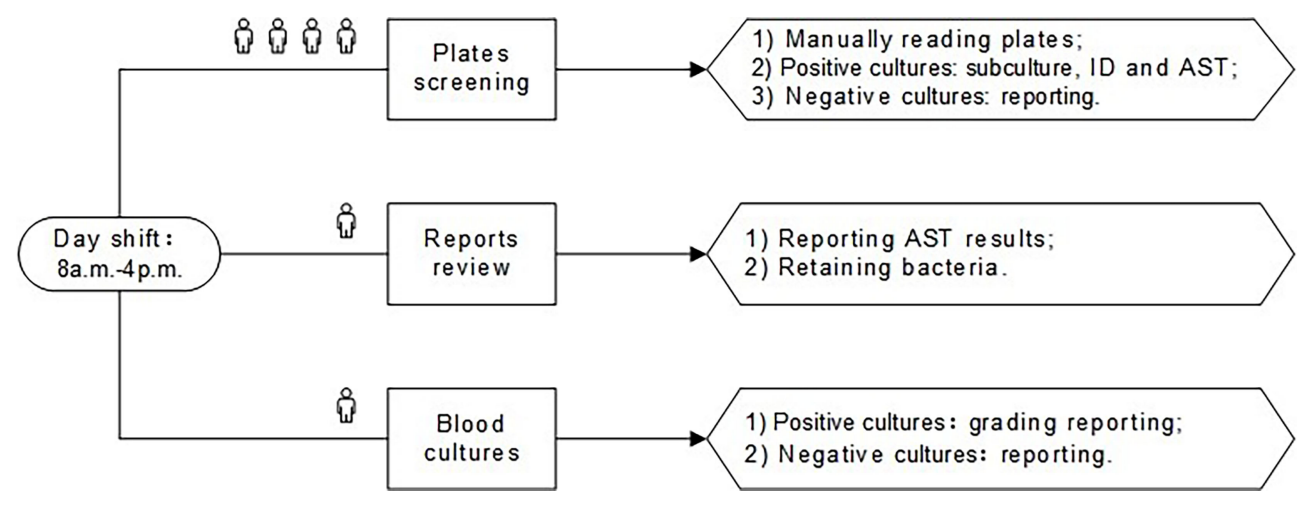

FIGURE 1 | The day shift of pre-TLA: four people for plates screening, one person for reports review, and one person for blood cultures. ID, identification; AST, antimicrobial susceptibility testing; TLA, total laboratory automation. 
AST reports review at 8 p.m. The three shifts post-TLA of our lab were elaborated in Figure 2.

\section{Laboratory Process Flow of CSF Specimens}

As with other types of specimens, when the system received sample information of CSF, the Kiestra automatically generated the corresponding plates: blood agar (BA) plates, chocolate agar (CA) plates, and thioglycollate broth. The CSF samples were handled semiautomated mode, and $50 \mu \mathrm{l}$ of CSF was inoculated separately on each plate or the broth, which was the same as pre-TLA. Then the plates were automatically put into the incubators and taken pictures at regular intervals. At 8 a.m. and 4.p.m., we read the images of the plates through the computer, and proceeded to the next step for the culturepositive specimens, while the culture-negative specimens were reported with negative results after 72 hours. At the same time, we would also observe the growth of broth. If the broth and the agar plates were positive, we directly performed ID and AST on the plates without further processing the broth. If the broth was positive and the agar plates were negative, we transferred the broth to the blood agar plate for subculture and then performed ID and AST after ruling out the possibility of contamination. The process flow of CSF specimens of our lab was elaborated in Figure 3.

\section{MALDI-TOF MS ID and AST}

Colonies were identified using the Bruker MALDI Biotyper instrument (Bruker Daltonik GmbH, Bremen, Germany). Species identification was considered valid if scores $\geq 2.0$. Scores $<1.7$ were regarded as unreliable; scores $\geq 1.7$ and $<2.0$ were considered as a reliable identification of genus.

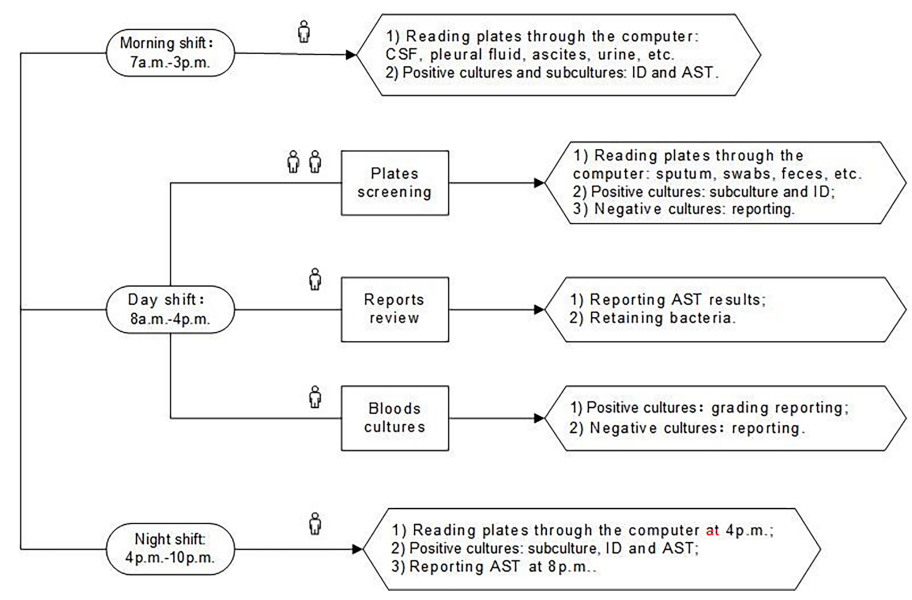

FIGURE 2 | The three shifts of post-TLA: one person for morning shift, four people for day shift, and one person for night shift. CSF, cerebrospinal fluid; ID, identification; AST, antimicrobial susceptibility testing; TLA, total laboratory automation.

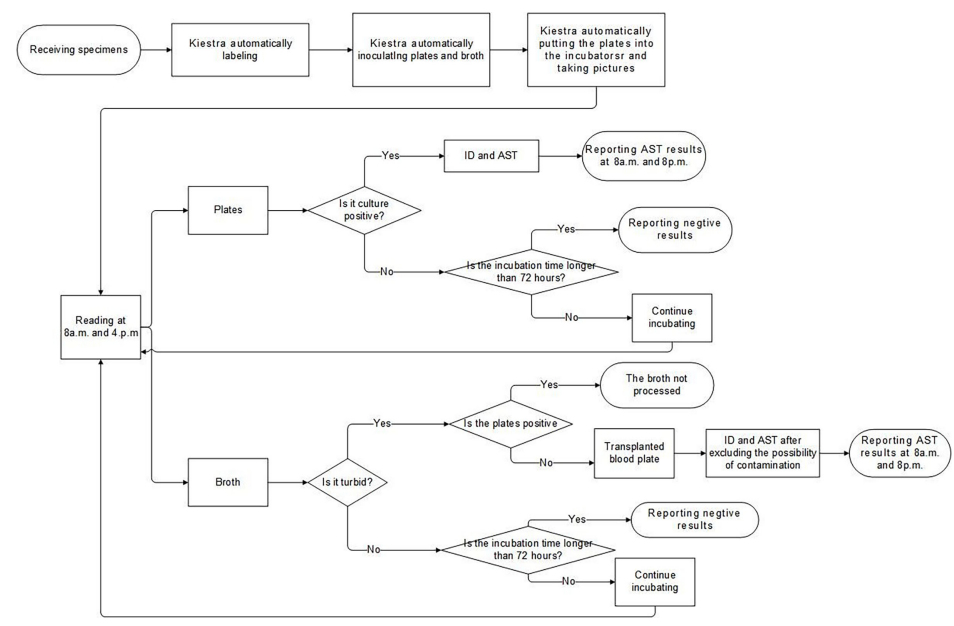

FIGURE 3 | The Laboratory process flow of CSF specimens. ID, identification; AST, antimicrobial susceptibility testing; CSF, cerebrospinal fluid. 
Colonies from primary cultures or subcultures were selected for AST by Kirby-Bauer disk diffusion (KB) (ThermoFisher, Massachusetts, USA) and Vitek II (BioMérieux, Montréal, France) as per CLSI (CLSI, 2019, 2020). For Vitek II susceptibility testing, colonies were picked and suspended in $0.45 \%$ saline. Turbidity of the inoculum was adjusted using the densitometer and the Vitek GN04 and GN67 AST cards were used for Gram-negative, Vitek GP67 for Gram-positive pathogens, or Vitek GP68 for Streptococcus pneumoniae, respectively. For KB testing, a $0.5 \mathrm{McF}$ arland standard inoculum was prepared by suspending isolated colonies from the primary BA plate incubated. Antibiotic disks were dispensed on the inoculated Mueller-Hinton agar (MHA) (Antubio, Zhengzhou, China) and incubated at $35^{\circ} \mathrm{C}$ according to CLSI testing and interpretation recommendations (CLSI, 2019, 2020).

\section{Data Extraction and Analysis}

The data including the time node of the sample reception, processed, and et al, was generated or recorded by our Laboratory Information System (Xinhe, Shanghai, China) spontaneously. We can export the detailed information, helping us to optimize laboratory management. To evaluate the impact of TLA on overall TAT, laboratory data for all cultures was extracted from our laboratory information system in two 6-month periods: preTLA (July to December 2019) and post-TLA (July to December 2020), respectively. During both periods, MALDI-TOF MS was used for the ID of isolates. TAT of positive cultures was defined as the time from specimen receipt by the laboratory to the final report. Comparisons of TAT in pre-TLA and post-TLA periods were analyzed using the Mann-Whitney $U$ test and Kruskal-Wallis tests, where applicable. All analyses performed are two-tailed, and $P$ values of $<0.05$ were considered significant.

\section{RESULTS}

\section{Comparison of the Time Needed From Receiving the Specimens to Final Reports Between Pre-TLA and Post-TLA Periods}

Figure 4 shows that there were two peaks of specimen reception starting at 9 a.m. and 5 p.m. After using Kiestra, the curve of the number of specimens received over time was flatter. Before TLA, the review of AST reports mostly started at 8 a.m. After using Kiestra, there was a small peak for AST report review at 8 p.m., because we scheduled the night shift.

\section{Comparison of TAT of Different Specimen Types Between Pre-TLA and Post-TLA Periods}

A total of 29199 positive-culture specimens were analyzed (14956 and 14243 from the pre-TLA and post-TLA periods, respectively) (Table 1 and Figure 5). For AST reports of all types of specimens, the median TAT for positive cultures decreased

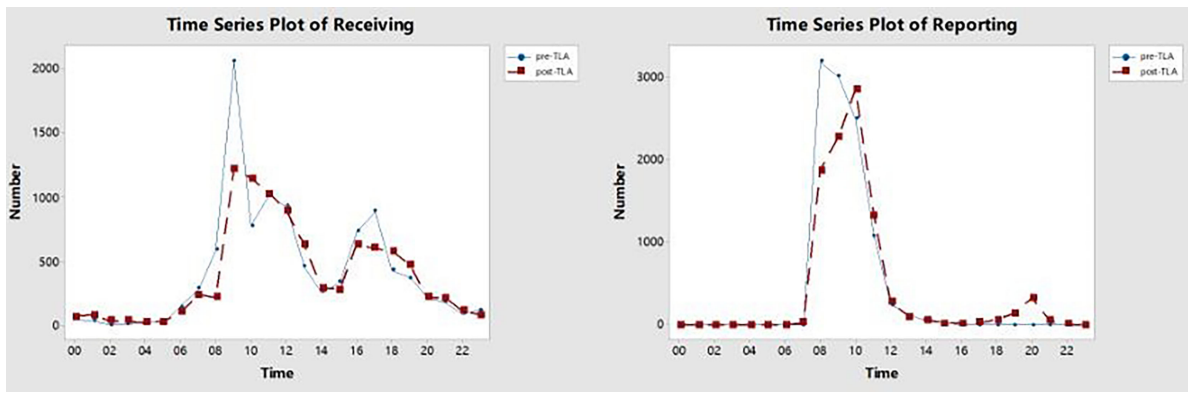

FIGURE 4 | The time series plot of receiving and reporting in pre-TLA and post-TLA. The X-axis represents the time in a 24-hour time display, and the Y-axis represents the number of receiving or reporting at each moment. TLA, total laboratory automation.

TABLE 1 | Comparison of TAT between pre-TLA and post-TLA.

\begin{tabular}{|c|c|c|c|c|c|}
\hline \multirow[t]{2}{*}{ Culture types } & \multicolumn{2}{|c|}{ No. of positive-culture (n) } & \multicolumn{2}{|c|}{ TAT (h) } & \multirow[t]{2}{*}{$P$-value } \\
\hline & Pre-TLA & Post-TLA & Pre-TLA & Post-TLA & \\
\hline Total & 68127 & 69126 & $65.93(47.91-86.30)$ & $63.53(46.85-77.37)$ & $<0.001$ \\
\hline Sputum & 7444 & 6312 & $64.38(47.00-86.50)$ & $61.41(46.03-79.08)$ & $<0.001$ \\
\hline Urine & 1772 & 2109 & $52.10(47.80-72.02)$ & $49.57(46.43-71.00)$ & $<0.001$ \\
\hline Blood & 2329 & 2408 & $68.49(60.72-87.43)$ & $66.60(58.99-85.17)$ & $<0.001$ \\
\hline CSF & 113 & 119 & $86.76(62.37-109.81)$ & $64.30(45.13-96.42)$ & 0.007 \\
\hline Ascites & 285 & 278 & $69.55(62.38-91.79)$ & $68.16(60.84-86.95)$ & 0.054 \\
\hline Pleural fluid & 97 & 107 & $68.83(61.05-92.44)$ & $69.97(46.79-91.55)$ & 0.325 \\
\hline
\end{tabular}

Results were shown as the median TAT in hours (with interquartile range in parentheses) and used the Mann-Whitney U test. TLA, total laboratory automation; TAT, turnaround time; CSF, cerebrospinal fluid. 


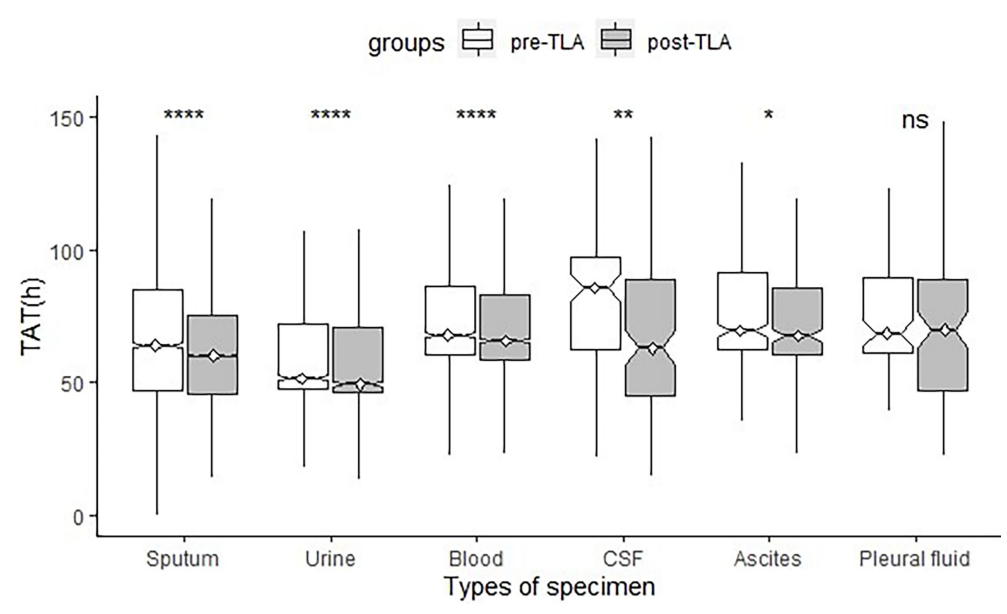

FIGURE 5 | Comparison of TAT of different specimen types between pre-TLA and post-TLA. ${ }^{\star \star \star \star} P<0.0001$; ${ }^{\star \star} P<0.01$; ${ }^{\star} P<0.1$; ns, no significance; TLA, total laboratory automation; TAT, turnaround time; CSF, cerebrospinal fluid.

significantly from pre-TLA to post-TLA (65.93 vs 63.53, $P<0.001)$. For different types of cultures, The TAT of CSF changed the most ( 86.76 vs 64.30, $P=0.007)$, followed by sputum (64.38 vs 61.41, $P<0.001$ ), urine (52.10 vs 49,57, $P<0.001$ ), blood (68.49 vs 66.60, $P<0.001)$. For Ascites and Pleural fluid, there was no significant difference $(P>0.05)$.

\section{Comparison of the Incidence of Broth Growth Only Between Pre-TLA and Post-TLA}

Further analysis found that the incidence of broth growth only for pre-TLA was $12.4 \%$ (14/133), while for post-TLA, it was 3.4\% (4/ 119). The difference was statistically significant $(P=0.01)$. When we excluded these specimens, the median TAT of pre-TLA reduced from 86.76 to 70.25 hours, while the median TAT of post-TLA reduced from 64.30 to 63.71 hours. The $P$-value estimating the difference between the two periods also changed from 0.007 to 0.013 .
Distribution of Strains Isolated From CSF

Figure 6 shows the distribution of strains isolated from CSF (only one strain was not shown). The common isolates of CSF specimens were Cryptococcus neoformans, coagulase-negative Staphylococcus, Acinetobacter baumannii, and Klebsiella pneumonia, which respectively accounted for $20.8 \%$, $18.5 \%$, $13.1 \%, 8.3 \%$.

\section{DISCUSSION}

We deeply modified the work organization of laboratory technicians and our schedule, which made our two compared study periods not strictly comparable because there were two variables: TLA and three shifts, but automation implementation in the lab need to perform these modifications in lab organization, notably reading time for the plates. Thanks to

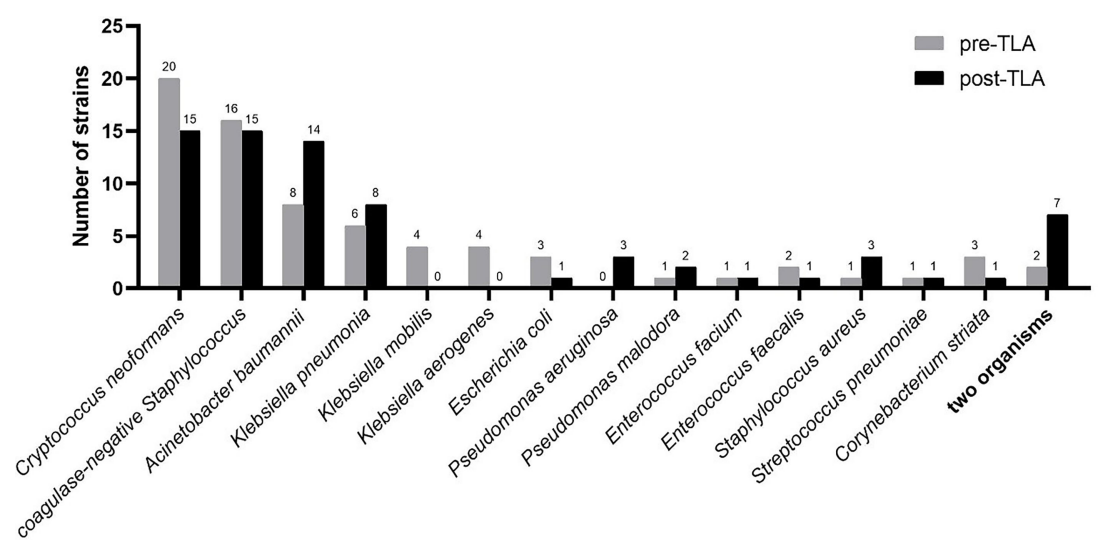

FIGURE 6 | Distribution of strains isolated from CSF in pre-TLA and post-TLA. CSF, cerebrospinal fluid; TLA, total laboratory automation. 
automation, it is quite easy to add additional reading time points as in our lab that plates were imaged every 6 hours. We did not reduce the number of laboratory technicians during the two periods. Thus, we modified the laboratory technicians' schedule to increase reading, ID, AST, etc. times and it was the combination of TLA and an extended reading time of laboratory technicians schedule that allowed a decrease of TAT. Automation helped to increase the duration of lab service without additional laboratory technicians. Therefore, like other studies, our lab also benefited from savings in labor cost (Culbreath et al., 2021; Zimmermann, 2021) and better control of the costs with reduced TAT thus resulting in a faster diagnosis (Leo et al., 2020). At the same time, reading the plates through the image can increase laboratory biosafety compared to reading the plates directly. Furthermore, if our lab was opened 24/7 with a reading service combined with machine learning (Bailey et al., 2019; Ford and McElvania, 2020; Culbreath et al., 2021), the TAT will perhaps more decrease.

Compared with the pre-TLA, the reception curve of the post-TLA was flatter, which was attributed to the fact that TLA can complete the tasks of receipt, streaking, and incubation of specimens in continuous or random mode rather than only in batch mode. Although this change may not be directly reflected in TAT, it will add the actual incubation time of the plate, increasing culture efficiency (Dauwalder et al., 2016; Graham et al., 2016). After adding a night shift, there was a small peak at 8 p.m. in the reporting curve of the post-TLA, which shortened the TAT by 12 hours and made the report available to clinicians in the next morning, so that the correct antibiotic treatment can be carried out in time (Graham et al., 2016).

Many studies have shown that TLA has the potential to reduce specimen processing time, optimize workflow, and decrease TAT (Mutters et al., 2014; Yarbrough et al., 2018; Theparee et al., 2018; Klein et al., 2018; Bailey and Burnham, 2019). In our study, TAT for all culture-positive specimens and different types of specimens was both reduced. Especially the TAT of CSF was shortened by about 22 hours, which was remarkable. Therefore, we carefully studied the process of our laboratory to determine the cause of the huge change in TAT. Further analysis found that the incidence of broth growth only in the pre-TLA period was significantly higher than that in the post-TLA period. When we excluded these specimens, the TAT difference between the two groups was reduced from 22 hours to less than 7 hours. According to the traditional laboratory process, when it was found that the plates did not grow but the broth grew, the broth was transferred to the plate until the bacteria grew on the plate before the next step of ID and AST, so the TAT could be as long as several days. Therefore, TLA has greatly improved the laboratory culture efficiency of sterile body fluids represented by CSF, enabling patients to receive timely antimicrobial treatment and reducing hospitalization costs and mortality.

Some scholars believe that enrichment broth cultures rarely contribute to the diagnosis of bacterial meningitis, and due to its high false-positive rate, positive CSF enrichment broth cultures contribute to diagnostic uncertainty (Chaudhry et al., 2011; Shah et al., 2012). This also reminds us that if TLA could greatly reduce the proportion of broth growth alone, maybe we can cancel broth enrichment culture, which certainly requires the support of larger experimental data combined with clinical practice.

The most common fungus isolated from CSF was Cryptococcus neoformans, and the most common bacteria were coagulasenegative Staphylococcus, Acinetobacter baumannii, and Klebsiella pneumonia, which was consistent with the results of other studies (Gonzalez et al., 2018; Chang et al., 2018; Tian et al., 2019). Some studies have reported that Alloscardivia omnicolens, Gardnerella vaginalis, Actinomyces spp., and Actinotignum schaalii were significantly more abundant in the urine samples incubated and processed with TLA (Klein et al., 2018), but we did not find similar pathogen spectrum changes in CSF samples.

In summary, using TLA and setting up three shifts shortened the TAT of our clinical microbiology laboratory, especially for CSF samples. One of the reasons is that the use of TLA increased the efficiency of culture, so the proportion of broth growth only was significantly reduced, which suggested the possibility that broth enrichment is not necessary when using TLA. Our research has some limitations. We conducted a retrospective study and derived data from Laboratory Information System, which may 1 present inaccurate results. Larger sample size will also be required for further research since the number of culturepositive samples isolated from CSF was relatively small in our current study. Finally, the "pre" and "post" timeframes of this study were also "pre" and "post" of COVID-19. it was difficult for us to assess whether and how much the COVID-19 affected the clinical microbiology laboratory process.

\section{DATA AVAILABILITY STATEMENT}

The datasets presented in this article are not readily available to comply with the Institutional data management requirements. Requests to access the datasets should be directed to Weili Zhang,648608511@qq.com.

\section{AUTHOR CONTRIBUTIONS}

WZ: Data analysis(lead); Writing-original draft (lead). SW and JD: Methodology (lead). QL, LX, LS, and YY: Methodology (supporting). YL and YLX: Data analysis (supporting). YM and MK: Writing-review \& editing (supporting). DL and YX: Writing-review \& editing (lead). All authors contributed to the article and approved the submitted version.

\section{FUNDING}

This work was supported by the Special Foundation for National Science and Technology Basic Research Program of China (Grant No. 2019FY101200). 


\section{REFERENCES}

Bailey, A. L., and Burnham, C. D. (2019). Reducing the Time Between Inoculation and First-Read of Urine Cultures Using Total Lab Automation Significantly Reduces Turn-Around-Time of Positive Culture Results With Minimal Loss of First-Read Sensitivity. Eur. J. Clin. Microbiol. Infect. Dis. 38, 1135-1141. doi: 10.1007/s10096-019-03512-3

Bailey, A. L., Ledeboer, N., and Burnham, C. D. (2019). Clinical Microbiology Is Growing Up: The Total Laboratory Automation Revolution. Clin. Chem. 65, 634-643. doi: 10.1373/clinchem.2017.274522

Chang, J. B., Wu, H., Wang, JH., Ma, B. T., Wang, R. Z., Wei, J. J., et al. (2018). Prevalence and Antibiotic Resistance of Bacteria Isolated From the Cerebrospinal Fluid of Neurosurgical Patients at Peking Union Medical College Hospital. Antimicrob. Resist. Infect. Control 7, 41. doi: 10.1186/ s13756-018-0323-3

Chaudhry, S. H., Wagstaff, D., Gupta, A., Bowler, I. C., and Webster, D. P. (2011). Enrichment Culture of CSF is of Limited Value in the Diagnosis of Neonatal Meningitis. Eur. J. Clin. Microbiol. Infect. Dis. 30, 931-933. doi: 10.1007/ s10096-011-1163-8

Croxatto, A., Dijkstra, K., Prod'hom, G., and Greub, G. (2015). Comparison of Inoculation With the InoqulA and WASP Automated Systems With Manual Inoculation. J. Clin. Microbiol. 53, 2298-2307. doi: 10.1128/JCM.03076-14

Croxatto, A., Prod'hom, G., Faverjon, F., Rochais, Y., and Greub, G. (2016). Laboratory Automation in Clinical Bacteriology: What System to Choose? Clin. Microbiol. Infect. 22, 217-235. doi: 10.1016/j.cmi.2015.09.030

Culbreath, K., Piwonka, H., Korver, J., and Noorbakhsh, M. (2021). Benefits Derived From Full Laboratory Automation in Microbiology: A Tale of Four Laboratories. J. Clin. Microbiol. 59(3):e01969-20. doi: 10.1128/JCM.01969-20

Dauwalder, O., Landrieve, L., Laurent, F., de Montclos, M., Vandenesch, F., Lina, G., et al. (2016). Does Bacteriology Laboratory Automation Reduce Time to Results and Increase Quality Management? Clin. Microbiol. Infect. 22, 236243. doi: 10.1016/j.cmi.2015.10.037

Ford, B. A., and McElvania, E. (2020). Machine Learning Takes Laboratory Automation to the Next Level. J. Clin. Microbiol. 58 (4), e00012-20. doi: 10.1128/JCM.00012-20

Gonzalez, S., Carbonaro, M., Fedullo, A. G., Sormani, M. I., Ceinos, M. D. C., Gonzalez, R., et al. (2018). Cerebrospinal Fluid Shunt-Associated Infections in Pediatrics: Analysis of the Epidemiology and Mortality Risk Factors. Arch. Argent Pediatr. 116, 198-203. doi: 10.5546/aap.2018.eng.198

Graham, M., Tilson, L., Streitberg, R., Hamblin, J., and Korman, T. M. (2016). Improved Standardization and Potential for Shortened Time to Results With BD Kiestra Total Laboratory Automation of Early Urine Cultures: A Prospective Comparison With Manual Processing. Diagn Microbiol. Infect. Dis. 86, 1-4. doi: 10.1016/j.diagmicrobio.2016.06.020

Klein, S., Nurjadi, D., Horner, S., Heeg, K., Zimmermann, S., Burckhardt, I., et al. (2018). Significant Increase in Cultivation of Gardnerella Vaginalis, Alloscardovia Omnicolens, Actinotignum Schaalii, and Actinomyces Spp. In Urine Samples With Total Laboratory Automation. Eur. J. Clin. Microbiol. Infect. Dis. 37, 1305-1311. doi: 10.1007/s10096-018-3250-6
Leo, S., Cherkaoui, A., Renzi, G., and Schrenzel, J. (2020). Mini Review: Clinical Routine Microbiology in the Era of Automation and Digital Health. Front. Cell Infect. Microbiol. 10, 582028. doi: 10.3389/fcimb.2020.582028

Mutters, N. T., Hodiamont, C. J., de Jong, M. D., Overmeijer, H. P., van den Boogaard, M., Visser, C. E., et al. (2014). Performance of Kiestra Total Laboratory Automation Combined With MS in Clinical Microbiology Practice. Ann. Lab. Med. 34, 111-117. doi: 10.3343/alm.2014.34.2.111

Shah, S. S., Hines, E. M., and McGowan, K. L. (2012). Cerebrospinal Fluid Enrichment Broth Cultures Rarely Contribute to the Diagnosis of Bacterial Meningitis. Pediatr. Infect. Dis. J. 31, 318-320. doi: 10.1097/INF. 0b013e318243e502

Theparee, T., Das, S., and Thomson, R. B.Jr. (2018). Total Laboratory Automation and Matrix-Assisted Laser Desorption Ionization-Time of Flight Mass Spectrometry Improve Turnaround Times in the Clinical Microbiology Laboratory: A Retrospective Analysis. J. Clin. Microbiol. 56(1):e01242-17. doi: 10.1128/JCM.01242-17

Thomson, R. B.Jr., and McElvania, E. (2019). Total Laboratory Automation: What Is Gained, What Is Lost, and Who Can Afford it? Clin. Lab. Med. 39, 371-389. doi: 10.1016/j.cll.2019.05.002

Tian, L., Zhang, Z., and Sun, Z. Y. (2019). Pathogen Analysis of Central Nervous System Infections in a Chinese Teaching Hospital From 2012-2018: A Laboratory-Based Retrospective Study. Curr. Med. Sci. 39, 449-454. doi: 10.1007/s11596-019-2058-7

Yarbrough, M. L., Lainhart, W., McMullen, A. R., Anderson, N. W., and Burnham, C. D. (2018). Impact of Total Laboratory Automation on Workflow and Specimen Processing Time for Culture of Urine Specimens. Eur. J. Clin. Microbiol. Infect. Dis. 37, 2405-2411. doi: 10.1007/s10096-018-3391-7

Zimmermann, S. (2021). Laboratory Automation in the Microbiology Laboratory: An Ongoing Journey, Not a Tale? J. Clin. Microbiol. 59 (3), e02592-20. doi: 10.1128/JCM.02592-20

Conflict of Interest: The authors declare that the research was conducted in the absence of any commercial or financial relationships that could be construed as a potential conflict of interest.

Publisher's Note: All claims expressed in this article are solely those of the authors and do not necessarily represent those of their affiliated organizations, or those of the publisher, the editors and the reviewers. Any product that may be evaluated in this article, or claim that may be made by its manufacturer, is not guaranteed or endorsed by the publisher.

Copyright (c) 2021 Zhang, Wu, Deng, Liao, Liu, Xiong, Shu, Yuan, Xiao, Ma, Kang, Li and Xie. This is an open-access article distributed under the terms of the Creative Commons Attribution License (CC BY). The use, distribution or reproduction in other forums is permitted, provided the original author(s) and the copyright owner(s) are credited and that the original publication in this journal is cited, in accordance with accepted academic practice. No use, distribution or reproduction is permitted which does not comply with these terms. 